\title{
Recurrence of Adult Granulosa Cell Tumor in the Greater Omentum 11 Years after Surgery
}

\author{
Mineto Ohta ${ }^{a, b}$ Yasuyuki Hara ${ }^{a}$ Toshiaki Kashiwadate ${ }^{a, b}$ \\ Masahiro Chin $^{a}$ Motohisa Hagiwara $^{a}$ Wataru Nakanishi ${ }^{b}$ Ken Ito ${ }^{a}$

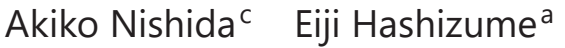 \\ aDepartment of Surgery, Nihonkai General Hospital, Yamagata, Japan; bepartment of \\ Gastroenterological Surgery, Graduate School of Medicine, Tohoku University, Sendai, \\ Japan; 'Department of Pathology, Nihonkai General Hospital, Yamagata, Japan
}

\section{Keywords}

Adult granulosa cell tumor · Greater omentum · Late recurrence · Undiagnosed intraperitoneal tumor

\begin{abstract}
Adult-type ovarian granulosa cell tumors (AGCTs) are very rare tumors that account for $<5 \%$ of all ovarian carcinomas. AGCTs have low malignancy potential and rarely metastasize 5-30 years after the initial diagnosis. Because time has passed from the first surgery and because recurrence develops in various locations, the differential diagnosis is difficult. In particular, tumors developing in the greater omentum are encountered rarely, and it is necessary to carefully consider the differential diagnosis, including primary and secondary neoplasms. Although $C T$ is useful to detect omental tumors, the diagnosis requires invasive procedures. We report a case of AGCT recurrence in the greater omentum that was resected during laparoscopic cholecystectomy. A patient visited our hospital with right-sided abdominal pain. The CT revealed gallbladder stones, a ureteral stone, and a right abdominal mass. The diagnosis of the abdominal tumor was difficult on the basis of blood biochemical testing, gastrointestinal endoscopy, or image inspection. Although the patient underwent several previous surgeries and there were no findings of malignancy with positron emission tomography, we chose to resect the tumor for combined diagnosis and treatment during laparoscopic cholecystectomy. Intraoperative findings showed that the tumor originated from the greater omentum, and the tumor was diagnosed as AGCT recurrence by pathology. A recurrence of AGCT in the greater omentum is very rare, and laparoscopic surgery was safe and useful for resection, in our case.
\end{abstract}




\section{Introduction}

Granulosa cell tumor (GCT) is a malignant tumor originating from sex cord stromal cells and which accounts for $2-5 \%$ of all ovarian carcinomas [1]. GCT is classified into adult GCT (AGCT) and juvenile GCT by clinical feature and histologic characteristics, and AGCT accounts for $95 \%$ of all cases. Approximately $22.5 \%$ of patients have no complaints [2], and most patients are diagnosed at stage 1 and have a favorable prognosis with surgical treatment [3, 4]. These tumors recur rarely 5-30 years after the first diagnosis [5]. Predicting recurrence is difficult [6], and several factors, such as tumor size, stage, and mitotic index, are associated with recurrence. The recurrence site differs depending on the clinical stage or surgical procedures [7]. Here, we report a case of recurrent AGCT in the greater omentum 11 years after the first surgery.

\section{Case Presentation}

A 58-year-old woman visited our emergency center with a complaint of right-sided back pain. CT examination showed gallstones, a right ureteral stone, and a right abdominal tumor. She had a history of several surgeries: appendectomy for appendicitis, simple hysterectomy for uterine myoma, salpingo-oophorectomy for a left ovarian tumor, and clipping for cerebral aneurysm at $17,47,48$, and 51 years of age, respectively. The current complaint occurred after the ureteral stone passed naturally. Preoperative inspection for gallstones and additional evaluation of the abdominal tumor were performed. Tumor markers, such as carcinoembryonic antigen, carbohydrate antigen 19-9, $\alpha$-fetoprotein, and soluble interleukin-2 receptor concentrations, were normal, and gastrointestinal endoscopy showed no abnormalities. The right abdominal tumor appeared as a submucosal tumor at the terminal ileum, with no findings of lymph node enlargement on CT (Fig. 1). There were also no accumulations of fluorine-18 fluorodeoxyglucose in the tumor with positron emission tomography imaging. We then decided to resect the tumor during cholecystectomy, to obtain a diagnosis. The surgery was performed via the 4-port laparoscopic-assisted method. After removing the gallbladder routinely, the abdominal cavity was examined. Preoperative imaging suggested that the tumor was present in the small-bowel mesentery; however, 2 tumors were actually present in the right distal greater omentum (Fig. 2a). We enlarged the umbilical camera port incision, which permitted extraction of the tumors (Fig. 2b). The tumors were well defined and were resected with sufficient margins (Fig. 3a). Pathological analysis revealed granulosa cells in the tumors (Fig. 3b), and the features were the same as for the left ovarian tumor resected 11 years earlier. The postoperative course was uneventful, and the patient was discharged 8 days after surgery.

\section{Discussion}

Tumors in the greater omentum are very rare [8]. Several tumor types, such as lipoma, leiomyosarcoma, solitary fibrous tumor, sarcoma, fibrosarcoma, desmoid tumor, fibroma, myxoma, infectious granulosa, and metastasis from known or occult cancer, have been reported [9]. Although CT is useful to detect omental tumors, diagnosis requires invasive procedures, such as biopsy or resection [10].

GCT is a malignant tumor originating from sex cord stromal cells and accounts for $2-5 \%$ of all ovarian cancers [1]. GCT is classified separately in adult or juvenile pattern by clinical presentation and histologic characteristics, and AGCT accounts for $95 \%$ of all cases. The most 
Ohta et al.: Rare Ovarian Tumor Recurred in the Greater Omentum 11 Years after First Surgery
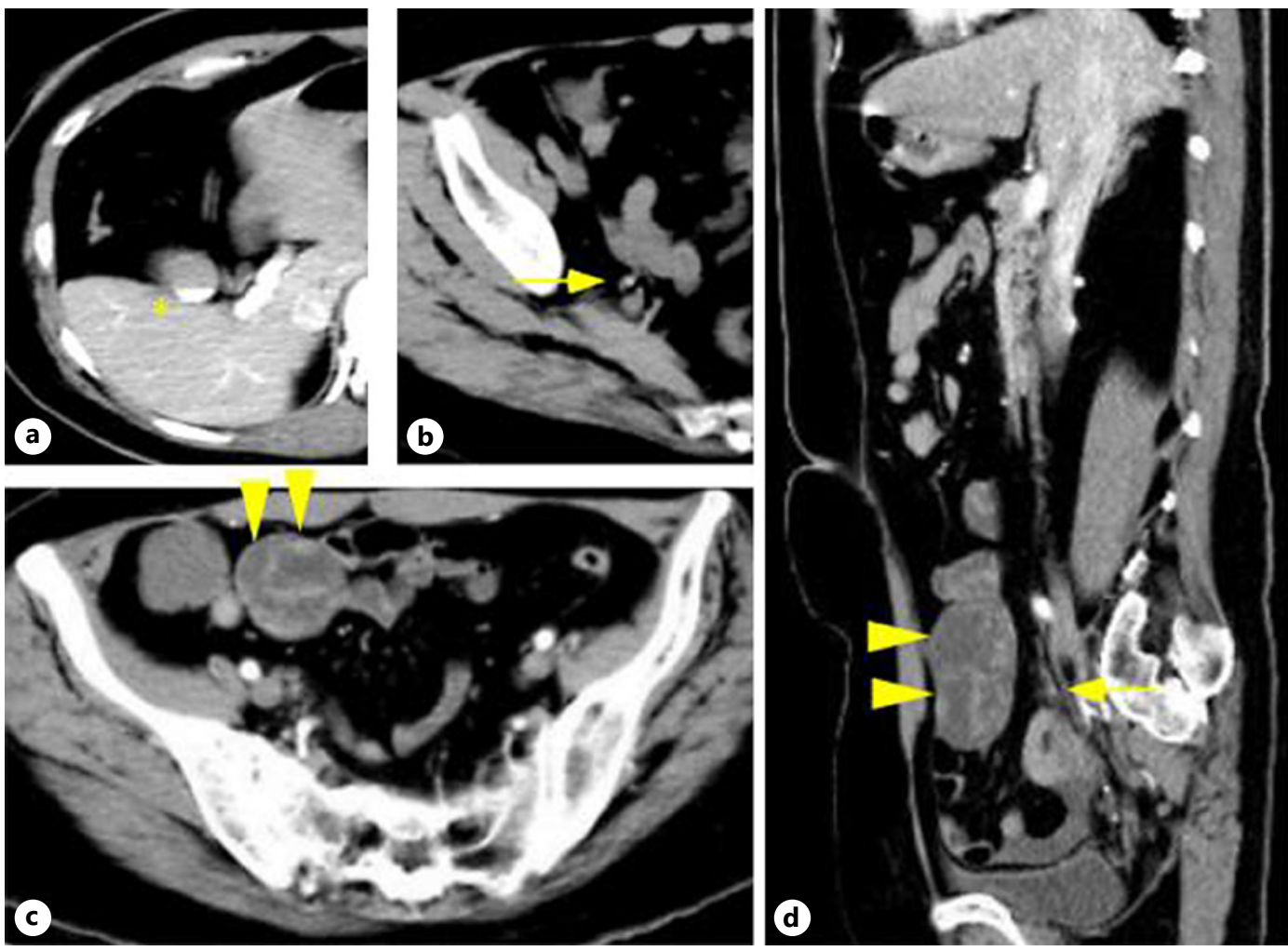

Fig. 1. CT images showing a right abdominal tumor with gallstones and a ureteral stone. CT imaging performed at arrival at our hospital showing gallstones (asterisk), a ureteral stone (yellow arrow), and a right abdominal tumor (yellow arrowheads). There were no findings of inflammation around the gallbladder. The CT value of the adipose tissue around the bladder stone was increased, which indicated inflammation and was the cause of the abdominal pain. The tumor in the lower right abdomen had a multicystic appearance and was located in the area of the distal ileum. From the CT imaging, the origin of the abdominal tumor was unclear, and the mass did not contact the small intestine or colon. CT axial views (a-c); CT sagittal view (d).

common symptoms are vaginal bleeding and a palpable abdominal mass; however, approximately $22.5 \%$ of patients have no complaints [2]. The majority of the patients (69.2-84.3\%) are diagnosed at stage 1 and have a favorable prognosis with surgical treatment. Five-year survival after surgery is $90.2-94.9 \%$, and surgical management of GCTs is decided on the basis of tumor stage [6]. Adjuvant therapy for stage 2-4 AGCT is controversial, and early and complete resection is supported by the best evidence [11]. Recently, the effectiveness of adjuvant chemotherapy has been reported [12], and surgery followed by chemotherapy may provide a good prognosis [3].

GCTs are rare and may recur 5-30 years after the initial diagnosis [6]. Several factors, such as tumor size, stage, and mitotic index, are associated with recurrence [3]. Recurrence sites vary, and the pelvis, ovary, mesentery, appendix, liver, and omentum have been reported [7]. Predicting recurrence and detecting metastatic sites are difficult $[13,14]$. Recently, micrometastasis of ovarian cancer to the omentum has been studied; however, omentectomy is controversial $[14,15]$.

In our case, left salpingo-oophorectomy was performed 11 years prior to the current tumor diagnosis, and AGCT was diagnosed by histopathology. Surveillance was completed soon after the surgery because the clinical stage was stage 1 . Because there was no evidence of malignancy in the preoperative evaluation for the current tumor, we chose laparoscopic 
Ohta et al.: Rare Ovarian Tumor Recurred in the Greater Omentum 11 Years after First Surgery
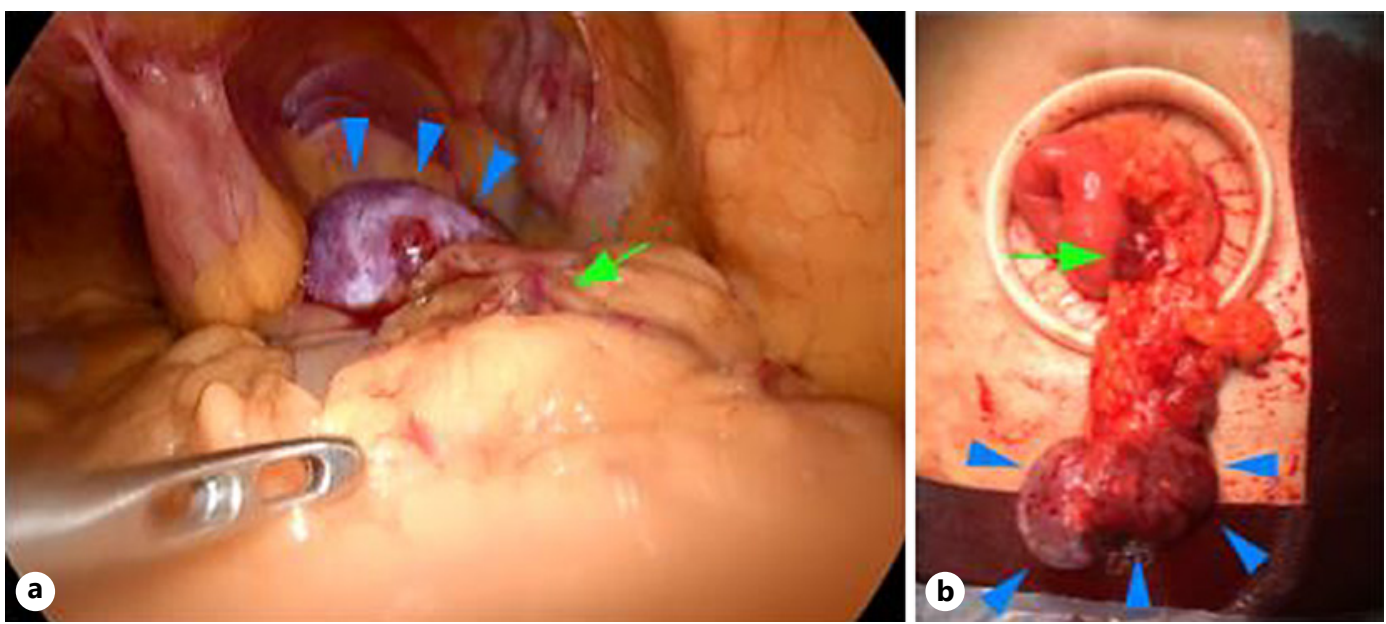

Fig. 2. Operative findings. a Laparoscopic view of the caudal pelvic region. The tumor was visible as a hemorrhagic and cystic lesion (blue arrowheads). Moreover, another tumor (green arrow) was found during examination of the entire abdominal cavity. b Extracorporeal extraction of the tumors. Two tumors (green arrow and blue arrowheads) are seen.

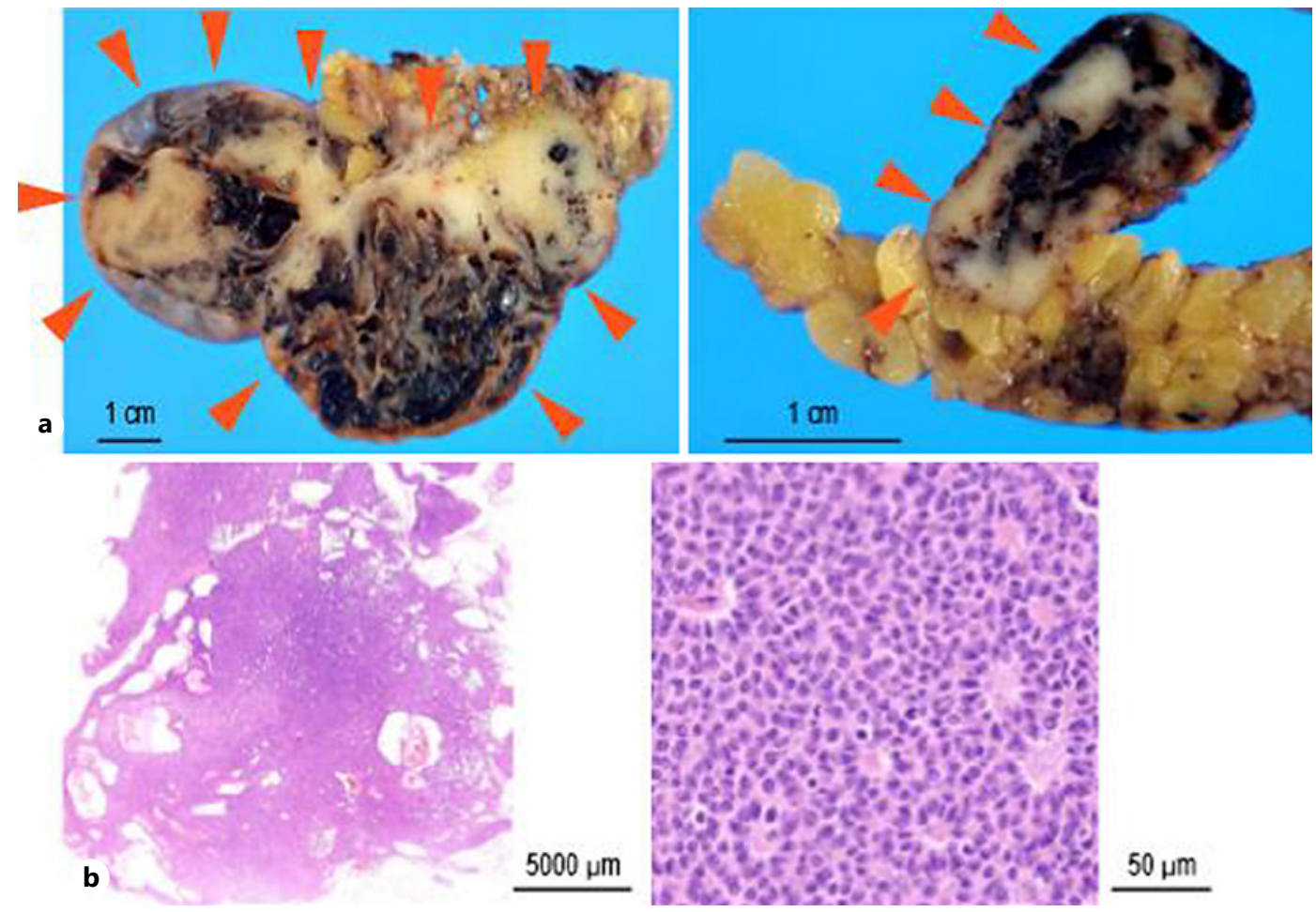

Fig. 3. Pathological findings. a Macroscopic findings of the resected tumors. The main tumor was a hemorrhagic and necrotic mass. The margin between the tumor and the fatty tissue of the greater omentum was clear. b Hematoxylin and eosin-stained microscopic images of both tumors. Tumor cells show scant cytoplasm with a coffee-bean-like nucleus. The pathological appearance was the same as that of the ovarian tumor resected 11 years earlier. 
tumor resection during cholecystectomy. The entire abdominal cavity could be observed using laparoscopy, and we successfully and safely resected 2 tumors. Laparoscopic surgery has been reported to be safe and useful for concurrent diagnosis and treatment [5].

In conclusion, we report a rare case of omental tumors originating from AGCT that was resected 11 years earlier. Even with early-stage AGCT, careful follow-up is required.

\section{Statement of Ethics}

The authors have no ethical conflicts to disclose. Written informed consent was obtained from the patient for publication of this case report and any accompanying images.

\section{Conflict of Interest Statement}

The authors declare no conflicts of interest.

\section{Funding Sources}

No funding was received for this article.

\section{Author Contributions}

All authors participated in the surgery or management of the patients. M.O. and T.K. prepared and performed the surgery. M.O. and Y.H. prepared the manuscript. The authors thank Jane Charbonneau, DVM, from Edanz Group (https://en-author-services.edanz.com/ ac) for editing a draft of this manuscript.

\section{References}

1 Fox H, Agrawal K, Langley FA. A clinicopathologic study of 92 cases of granulosa cell tumor of the ovary with special reference to the factors influencing prognosis. Cancer. 1975 Jan;35(1):231-41.

2 Lee IH, Choi CH, Hong DG, Song JY, Kim YJ, Kim KT, et al. Clinicopathologic characteristics of granulosa cell tumors of the ovary: a multicenter retrospective study. J Gynecol Oncol. 2011 Sep;22(3):188-95.

3 Ertas IE, Gungorduk K, Taskin S, Akman L, Ozdemir A, Goklu R, et al. Prognostic predictors and spread patterns in adult ovarian granulosa cell tumors: a multicenter long-term follow-up study of 108 patients. Int J Clin Oncol. 2014 Oct;19(5):912-20.

4 Khosla D, Dimri K, Pandey AK, Mahajan R, Trehan R. Ovarian granulosa cell tumor: clinical features, treatment, outcome, and prognostic factors. N Am J Med Sci. 2014 Oct;6(5):133-8.

5 Malmström H, Högberg T, Risberg B, Simonsen E. Granulosa cell tumors of the ovary: prognostic factors and outcome. Gynecol Oncol. 1994 Jan;52(1):50-5.

6 Thomakos N, Biliatis I, Koutroumpa I, Sotiropoulou M, Bamias A, Liontos M, et al. Prognostic factors for recurrence in early stage adult granulosa cell tumor of the ovary. Arch Gynecol Obstet. 2016 Nov;294(5):1031-6.

7 Ud Din N, Kayani N. Recurrence of adult granulosa cell tumor of the ovary: experience at a tertiary care center. Ann Diagn Pathol. 2014 Jun;18(3):125-8.

8 Ishida H, Ishida J. Primary tumours of the greater omentum. Eur Radiol. 1998;8(9):1598-601.

9 Susan W, Robert E, Paul M, Raymond S. The greater omentum. AJR Am J Roentgenol. 1997 Mar;168(3):683-7.

10 Pérez Montilla ME, Lombardo Galera S, Espejo Herrero JJ, Sastoque JM, Zurera Tendero L. Diagnostic performance of imaging-guided core needle biopsy of the mesentery and peritoneum. Radiología. 2018;60(2):12835.

11 Sun HD, Lin H, Jao MS, Wang KL, Liou wS, Hung YC, et al. A long-term follow-up study of 176 cases with adulttype ovarian granulosa cell tumors. Gynecol Oncol. 2012 Feb;124(2):244-9. 
12 Pautier P, Gutierrez-Bonnaire M, Rey A, Sillet-Bach I, Chevreau C, Kerbrat P, et al. Combination of bleomycin, etoposide, and cisplatin for the treatment of advanced ovarian granulosa cell tumors. Int J Gynecol Cancer. May-Jun 2008;18(3):446-52.

13 Chen YH, Chang LC, Soong RS. A late recurring and easily forgotten tumor: ovarian granulosa cell tumor. World J Surg Oncol. 2012 May 16;10:85.

14 Bayrak M, Yılmaz A, Yılmaz F, İlhan 0, Oz Atalay F, Ozan H. Omental micrometastasis in endometrial cancer. Oncol Res Treat. 2019;42(9):466-9.

15 Fujita F, Eguchi S, Takatsuki M, Kobayashi K, Kanetaka K, Ito M, et al. A recurrent granulosa cell tumor of the ovary 25 years after the initial diagnosis: a case report. Int J Surg Case Rep. 2015;12:7-10. 